\title{
Nanosynthesis of Tunable Composite Materials by Room-Temperature Pulsed Focused Electron Beam Induced Chemical Vapour Deposition
}

\author{
M. S. Gabureac, L. Bernau, and I. Utke* \\ Swiss Federal Laboratories for Materials Science and Technology (EMPA), Feuerwerkerstr. 39, \\ CH-3602 Thun, Switzerland
}

\begin{abstract}
Hydrocarbons inherently present in standard high-vacuum scanning electron microscopes can be favorably used for co-deposition with functional molecules injected into the chamber. By varying the beam exposure pulse time the carbon content incorporated into the deposit can be tuned. In the particular case when the hydrocarbons are provided by surface diffusion, the composition depends also on the size of the final deposits. This dependency can be used as an additional parameter, besides the beam pulse time, in order to tune the metal/matrix ratio and to obtain new nanoscale materials with tailored physical properties. We present and discuss experimental results on composition tunability by pulsed electron-beam deposition for the two-adsorbate system $\mathrm{Co}_{2}(\mathrm{CO})_{8}$ /hydrocarbon and their use in fabricating Hall nanosensors of cobalt-carbon nanocomposite material with enhanced magnetic sensitivity and high magnetic spatial resolution.
\end{abstract}

Keywords: Pulsed Focused Electron Beam Induced Deposition, Carbon Co-Deposition, Composition Tuning, Nanocomposite Magnetic Nanosensors.

\section{INTRODUCTION}

The gas assisted focused electron or ion beam (FEB/FIB) induced deposition is a direct write maskless deposition technique that can produce in a single step ultra high resolution nano-structures or 3D nanodevices inside standard scanning electron microscopes (SEM) equipped with gas injection systems (GIS). ${ }^{1-5}$ It converts a well-established observation tool to an innovative nanofabrication workbench for nanodevices in the fields of photonics, biology, and electronics. When combined with the throughput of the next generation multi beam machines, it promises to provide a viable route to cope with the increasingly demands for maskless nano-structuring methods at highest lateral resolution (sub-10-nm) and maximum flexibility in shape and material. The synthesis is performed predominantly within the spot size of a few nanometers and does not affect other sensitive parts of the nanodevices. ${ }^{6}$ Materials synthesized by focused electron and ion beams were mostly confined to applications as electrically conducting wires (metal deposits), hard scanning probe tips (diamond like material), and

\footnotetext{
*Author to whom correspondence should be addressed
}

optically transparent material (silicon oxide). ${ }^{2}$ Depending on the metal:matrix ratio and the matrix conductivity, FEB/FIB-nanowires with outstanding properties have been recently nano-synthesized featuring superconductivity ${ }^{7,8}$ piezoresistivity $^{9,10}$ superparamagnetism ${ }^{11}$ but also ferromagnetism $^{12,13}$ when the metal concentration is higher than a certain threshold.

FEBID benefits from a similar broad choice of precursors as CVD. However, the difference is provided by the high resolution local deposition, since the molecule decomposition is due to electron-triggered dissociation rather than thermal dissociation, thus keeping the reaction confined to the size of the electron beam and the active electron interaction volume.

Although the electron induced deposition of high purity metals is sometimes possible (apparently depending on the autocatalytic properties of the deposited metals), ${ }^{1,14-16}$ in general, the result is a nanocomposite material, consisting of a mixture of the desired metal nanoparticles surrounded by a matrix of different composition. This stems from different sources, e.g., fragments of ligands from the original precursor molecule, co-deposition of carbon from background vacuum, or even contamination adsorbates from the substrate. ${ }^{17}$ 
Despite the fact that the co-deposition of carbon during FEBID is a nuisance for pure deposits, the inherent hydrocarbon sources in high-vacuum microscope chambers have been successfully used for producing carbon masks ${ }^{18,19}$ and, more recently, for the tuning of the metal-carbon ratio that allowed increasing the magnetic sensitivity of $\mathrm{Co}-\mathrm{C}$ nano-Hall sensors. ${ }^{11}$

\section{COMPOSITION TUNING USING THE TWO ADSORBATE CONCEPT}

Recently, we demonstrated that it was possible to control the composition of $\mathrm{Co}-\mathrm{C}$ FEB deposits by varying the beam exposure pulse time and proposed an analytical two adsorbate model that describes the tuning of the composition for any two co-injected molecules. ${ }^{20}$ This general concept predicts the pulse time range within which variation in composition can be realized by the selective depletion of the adsorbates. For sufficiently high electron fluxes $f$, the ultimate tunability is determined solely by the ratio $\sigma_{A} \tau_{A} / \sigma_{B} \tau_{B}$ where $\sigma$ is the electron induced dissociation cross section and $\tau$ is the residence time of the adsorbates $A$ and $B$. In Figure 1 we illustrate the predictions of the two adsorbate concept for a particular case when

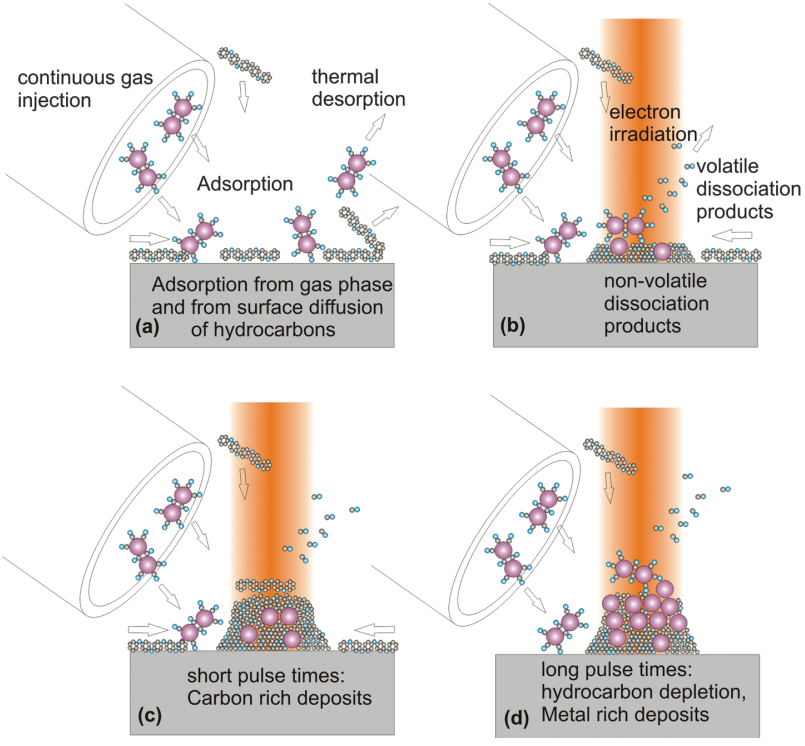

Fig. 1. Composition tuning based on selective depletion of for $\mathrm{Co}_{2}(\mathrm{CO})_{8}$ and hydrocarbon. (a) Before irradiation, competitive adsorption from the gas phase leads to an equilibrium surface density of injected molecules and background hydrocarbon molecules $\left(\approx 10^{3}: 1\right)$. (b) Upon electron irradiation, hydrocarbons are quickly depleted due to their larger electron-impact dissociation cross-section. The liberated surface sites are preferentially filled by $\mathrm{Co}_{2}(\mathrm{CO})_{8}$ molecules due to their larger partial pressure and longer residence time. A new, $\mathrm{Co}_{2}(\mathrm{CO})_{8}$-rich adsorbate equilibrium $\left(\approx 5 \times 10^{4}: 1\right)$ establishes during exposure. (c) If electron exposure now continues (>100 ns) a metal-rich deposit is obtained as long as the Co is still in the electron limited regime. (d) If exposure is interrupted (for $\approx 10 \mathrm{~ms}$ ), the initial adsorbate equilibrium re-establishes leading to a carbon-rich deposit upon the next exposure pulse.

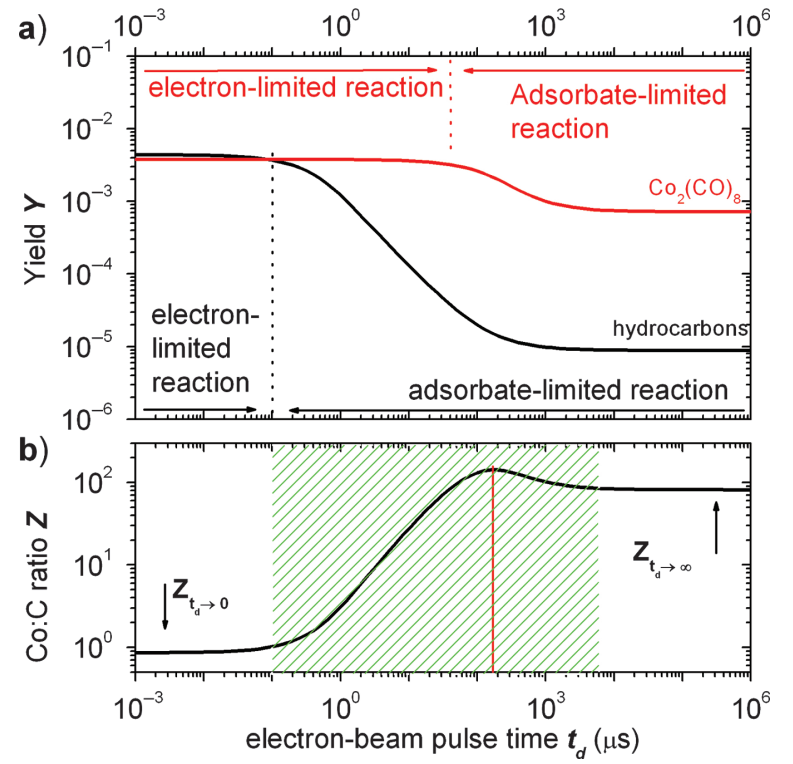

Fig. 2. Calculated dissociation reaction yields $Y$ (per primary electron) for the two adsorbate species that lead to an exposure window for the yield ratio $Z$ where the composition can be tuned. ${ }^{20}$

one adsorbate is provided by a gas injection system while the other stems from the hydrocarbon molecules present in the deposition chamber background gas. We purposely discard the influence of water vapour for reasons explained in Ref. [21].

In contrast to classical chemical vapour deposition exploiting co-evaporation, the deposit will be locally confined and the composition will not only depend on the ratio of molecule flow but rather on the electron-impact dissociation efficiency of each individual adsorbate species giving a further degree of freedom to the control of tuned material synthesis.

The composition of the deposit is given by the yield ratio $Z$ of the two adsorbates as explained in Ref. [20] and can be tuned between the limits:

$$
Z_{t_{d \rightarrow 0}} / Z_{t_{d} \rightarrow \infty}=\left(1 / f+\sigma_{A} \tau_{A}\right) /\left(1 / f+\sigma_{B} \tau_{B}\right)
$$

where $f$ is the primary electron flux and $t_{d}$ the pulse time.

As shown in Figure 2, this leads to an exposure window where composition can be tuned, with a peak for the maximum $\mathrm{Co}: \mathrm{C}$ ratio above the level established at large pulse times $\left(Z\left(t_{d} \rightarrow \infty\right)\right)$.

\section{PULSED FEB DEPOSITION OF SUB-MICRON PLANAR STRUCTURES}

The FEBID experiments were done in a Hitachi S3600 vacuum chamber equipped with tungsten thermal emission filament. The pumping system consists of an oil diffusion pump back-pumped by an oil roughing pump. This results in a base pressure of a few $10^{-5}$ mbar with a particular fingerprint of hydrocarbons in the background vacuum 
resulting in an impinging flux of $7.5 \cdot 10^{14} \mathrm{~cm}^{-2} \mathrm{~s}^{-1}$ hydrocarbons. The $\mathrm{Co}_{2}(\mathrm{CO})_{8}$ was supplied via an Alemnis gas injection system. A detailed description of the exact experimental conditions can be found in Refs. [20 and 21].

\subsection{Carbon Deposition from Vacuum Background}

The carbon contamination inside the SEM chamber during FEBID stems from two different sources. The contamination from residual gases from the background vacuum and/or from unclean substrates ${ }^{17}$ could be avoided by working under UHV conditions. However, the contamination arising from the incomplete decomposition of precursor adsorbates or from the co-deposition of ligand fragments when using organo-metallic precursors ${ }^{2}$ are sometimes inherent processes that can become important regardless of the background pressure; especially, when mechanisms such as the chemisorption of $\mathrm{CO}$ on the surface of the transition metals are involved. ${ }^{22}$

In Figure 3 we show the hydrocarbon deposition rates for $(600 \mathrm{~nm})^{2}$ squares at two different microscope background pressures showing the importance of the base pressure for any FEBID experiment and the amount of co-deposited carbon during standard FEBID processes as shown in Figure 1.

While FEBID in UHV on cleaned substrates allows for the elimination of surrounding contamination sources, the contamination which arises from the elements included in the precursor itself is still present and the deposits from organo-metallic precursors such as $\mathrm{Fe}(\mathrm{CO})_{5}$ can still include carbon. ${ }^{23,24}$ The mechanisms for the decomposition of $\mathrm{CO}$ ligands by electrons have been studied intensely studied in UHV. ${ }^{22,25}$

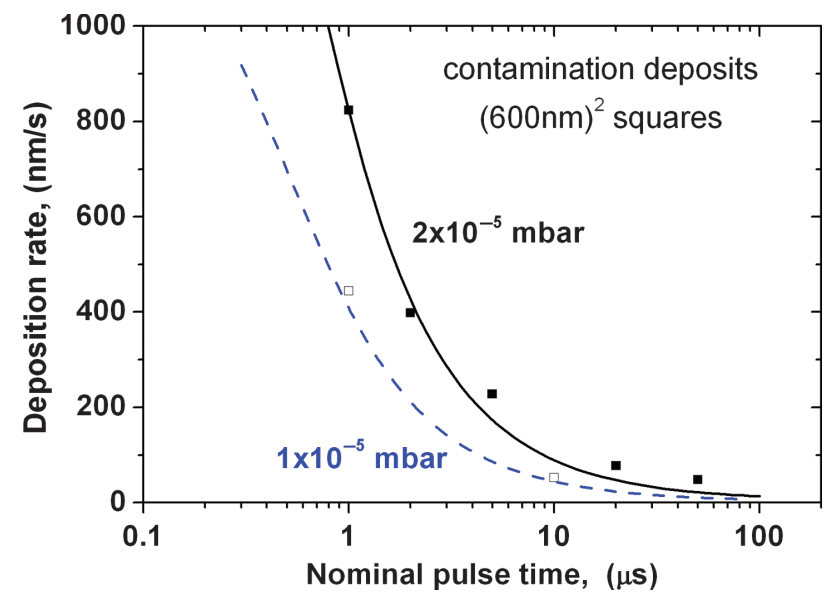

Fig. 3. Experimental values and single-adsorbate model fits of the vertical deposition rate according to equation S3 from Ref. [20] in the depletion regime for $(600 \mathrm{~nm})^{2}$ square hydrocarbon deposits at 1 and $2 \cdot 10^{-5}$ mbar base pressure. The total electron dose was maintained constant at $10 \mathrm{C} / \mathrm{cm}^{2}$.

\subsection{Surface Diffusion of Hydrocarbons}

It is known that the surface diffusion is a mechanism that can play an important role in the adsorbate supply during FEBID. ${ }^{2}$ In the dual adsorbate model presented in Ref. [20] we took this mechanism into account by an effective (size dependent) residence time, which allowed for deriving analytical solutions for the system of first order differential equations describing the competitive adsorption and to derive general observations for the tunability of the co-deposition process, see Eq. (1).

In order to estimate the importance of the hydrocarbon surface diffusion we have measured the height profiles of the $3 \mu \mathrm{m}$ square deposits as shown in Figure 4.

The deposits were made using identical parameters (10 $\mu$ s dwell time, total dose of $10 \mathrm{C} / \mathrm{cm}^{2}, 1 \mathrm{nA}$ current, $25 \mathrm{kV}$ acceleration voltage, $30 \mathrm{~nm}$ pitch and spiral writing strategy as explained in Ref. [20]) and a base pressure of $1 \times 10^{-5}$ mbar. Clearly, at the edges, the deposition of carbon is diffusion enhanced as one obtains a profile typical for this deposition regime. ${ }^{26,27}$ From the rim of the AFM profile it appears that the diffusion length of the hydrocarbons is about $1 \mu \mathrm{m}$ under the experimental conditions used. The deposit becomes thinner towards the center. Since the hydrocarbon adsorbates were first deposited on the edge of the square deposit they cannot contribute by surface diffusion anymore; the deposition rate at the deposit centre is limited by the gas transport of hydrocarbons (about $25 \%$ of the diffusion enhanced rate). This allows us to establish quasi single adsorbate conditions for the $\mathrm{Co}_{2}(\mathrm{CO})_{8}$ molecule and to study its dissociation under electron exposure. Since $\mathrm{Co}_{2}(\mathrm{CO})_{8}$ is abundantly injected via gas phase the centre is almost flat. The surface diffusion contribution of the hydrocarbon is largely suppressed by competitive adsorption as only faint rim formation is observed (5\% difference to centre level).

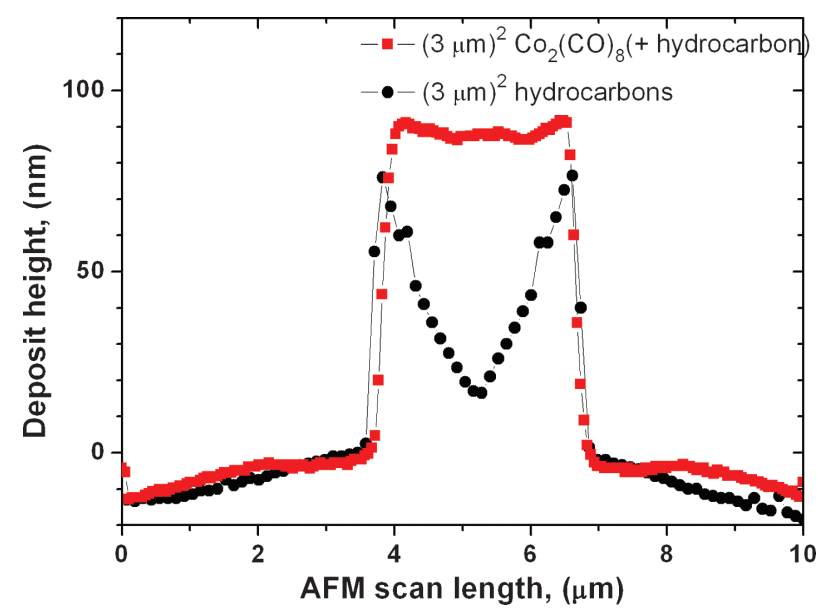

Fig. 4. AFM profile of $3 \mu \mathrm{m}$ square deposits of $\mathrm{Co}_{2}(\mathrm{CO})_{8}$ (black squares) and hydrocarbons (blue squares) from Ref. [21].

J. Nanosci. Nanotechnol. 11, 7982-7987, 2011 


\subsection{Composition Tuning by Pulse Time}

We first discuss the results obtained under quasi singleadsorbate conditions (3 $\mu \mathrm{m}$ squares) and varying pulse time before considering the two-adsorbate system. The electron induced dissociation of adsorbed cobalt carbonyl gives a deposit composition of $\mathrm{Co}_{2} \mathrm{C}_{0.6} \mathrm{O}_{0.4}$ regardless of electron beam pulse time, see Figure 5 (always measured in the centre of the deposit). Comparing with the intact adsorbate stoichiometry $\mathrm{Co}_{2} \mathrm{C}_{8} \mathrm{O}_{8}$, almost all carbonyls were removed from the cobalt atom. Only a fraction of carbon and oxygen is found in the electron-induced deposit. Together with the constant $\mathrm{Co}$ and $\mathrm{C}$ content this implies that incomplete adsorbate decomposition is not a mechanism for the carbon co-deposition in our system. The hydrocarbon deposit obtained under single adsorbate conditions had a constant composition of $\mathrm{C}_{0.89} \mathrm{O}_{0.11}$ similar to Ref. [28]. Such a composition could be obtained by dissociating the hydrogen from octanol, $\mathrm{C}_{8} \mathrm{H}_{17} \mathrm{OH}$, which we have chosen as model hydrocarbon molecule in Ref. [20].

Two adsorbate conditions prevail in the $600 \mathrm{~nm}$ square deposits. Figure 5 summarizes the EDX results for planar squares with lateral dimensions of $600 \mathrm{~nm}$ and $3 \mu \mathrm{m}$. The beam current used was of $1 \mathrm{nA}$ and the total dose was of $10 \mathrm{C} / \mathrm{cm}^{2}$. The refresh time was larger than $10 \mathrm{~ms}$ allowing for the full replenishment of the $\mathrm{Co}_{2}(\mathrm{CO})_{8}$ precursor adsorbates.

The deposition rates calculated from the heights measured by AFM and then fitted with single adsorbate models, allowed to obtain the values for the electron-impact dissociation cross-section $\sigma$ and the surface residence time $\tau$, for the $600 \mathrm{~nm}$ squares used for the composition tuning, as described in Ref. [20]. Because of hydrocarbon carbon surface diffusion, these are effective values linked to the lateral size of the deposit.

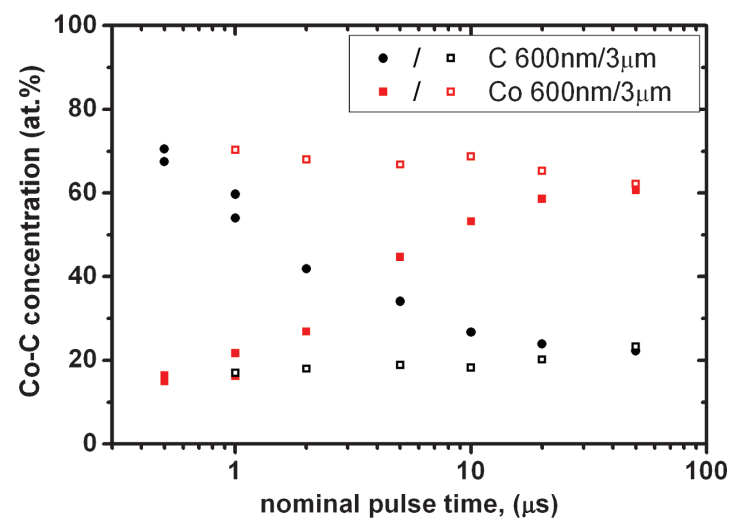

Fig. 5. Variation of Co and $\mathrm{C}$ content for FEB nano-deposits versus the beam pulse time. The $3 \mu \mathrm{m}$ squares represent quasi single adsorbate conditions. The $\mathrm{Co}$ and $\mathrm{C}$ concentration variation in the $600 \mathrm{~nm}$ squares for the $500 \mathrm{~ns}-100 \mu$ s pulse time interval is consistent with the two-adsorbate model described in Ref. [20]. The oxygen level (not shown) was constant around 15 at.\% (within the experimental precision of \pm 5 at.\%).

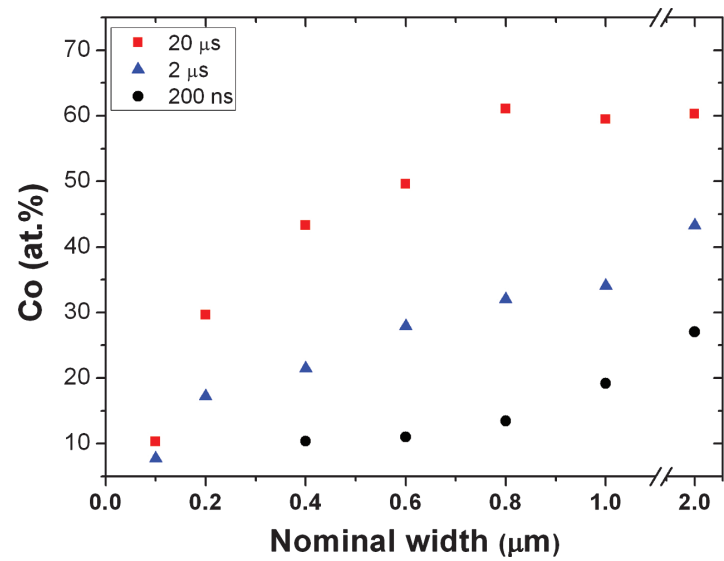

Fig. 6. Cobalt concentration in FEBID square deposits with varying dimensions for three pulse times.

\subsection{Composition Tuning by Size Variation}

An unveiled consequence of the carbon co-deposition mechanism via surface diffusion is the fact that the composition will change with deposit size. This is shown in Figure 6, where we have deposited squares with different sizes. The metal concentration at the centre of the square increased linearly with the width. We have used a $25 \mathrm{kV}, 10 \mathrm{nA}$, beam current (beam size approximately $200 \mathrm{~nm}$ ) and 3 different pulse times per pixel. The deposits were done at $1 \times 10^{-5}$ mbar background pressure on a $200 \mathrm{~nm}$ thick Au layer, evaporated on a Si wafer on top of a thermally grown $\mathrm{SiO}_{2}$ layer. The total dose per pixel was fixed to $5 \mathrm{C} / \mathrm{cm}^{2}$ and the distance between consecutive pixels was $4 \mathrm{~nm}$. The scan was a spiral fill allowing for the full replenishment with the $\mathrm{Co}_{2}(\mathrm{CO})_{8}$ between consecutive exposures (more than $100 \mathrm{~ms}$ refresh time). Both the pulse time and the refresh time were fixed for all deposits, as well as the number of pattern repetitions. For each pulse time the only parameter that varied was the square size.

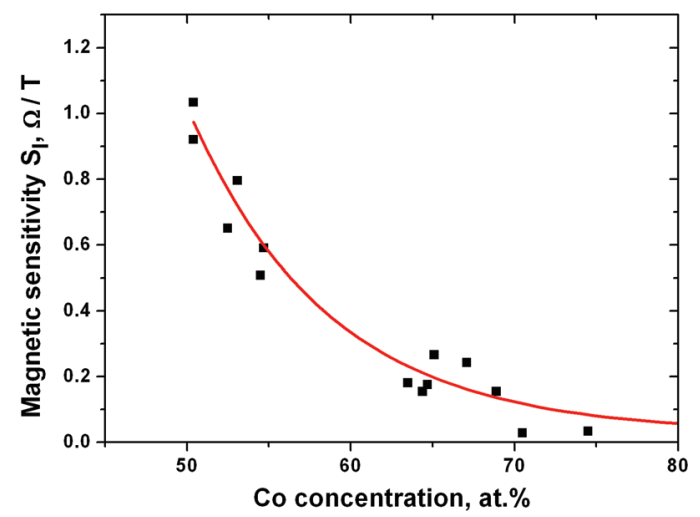

Fig. 7. Exponential increase in the magnetic field sensitivity when the Co concentration is approaching the metal-insulator transition as explained in Refs. [11 and 29]. 
Table I. Magnetic properties for different types of sensors from Ref. [11]: although the magnetic sensitivity $\left(S_{l}\right)$ and field resolution $\left(B_{\text {min }}\right)$ of the Co-C deposits are small, the magnetic spatial resolution $\Phi_{\min } / \Phi_{0}$ is the best; $\Phi_{0}=h /(2 e)$ is the magnetic flux quantum.

\begin{tabular}{|c|c|c|c|c|c|c|c|c|c|}
\hline Sensor type & InAs & InGaAs & $n-\mathrm{Si}$ & Co & $\mathrm{FePt}$ & $\mathrm{Bi}$ & $\mathrm{InSb}$ & InAsSb & $\mathrm{Co}-\mathrm{C}$ \\
\hline Width (nm) & 1000 & 2000 & 2400 & 100 & 160 & 50 & 500 & 1000 & 100 \\
\hline$S_{I}(\Omega / T)$ & 616 & 700 & 175 & 0.03 & 325 & 4 & - & 2750 & 0.15 \\
\hline$B_{\min }\left(\mathrm{nT} / \mathrm{Hz}^{1 / 2}\right)$ & 4300 & 400 & 200 & 50000 & 166000 & 80000 & 720 & 51 & 900 \\
\hline$\Phi_{\min } / \Phi_{0}$ & $2 \times 10^{-3}$ & $2 \times 10^{-4}$ & $5.5 \times 10^{-4}$ & $2.5 \times 10^{-4}$ & $1.6 \times 10^{-4}$ & $10^{-4}$ & $9 \times 10^{-5}$ & $2.5 \times 10^{-5}$ & $5 \times 10^{-6}$ \\
\hline
\end{tabular}

The increase in cobalt content with increasing width can be explained as follows: once the carbon was depleted by long pulse exposure along the edges of the squares, the amount of carbon arriving in the middle of the deposition area by surface diffusion diminishes. This hidden size dependence mechanism was accounted for in Ref. [20] where $\tau$ and $\sigma$ are effective values obtained from the fitting of the deposition rates of specific square dimensions with the single adsorbate model.

It is also important to note that the composition depends intrinsically on the beam flux (current density) as can be seen in Eq. (1). As a consequence, a different beam flux will move the exposure window for the composition tunability (see Fig. 2) with respect to the electron beam pulse time.

The co-deposition of $\mathrm{C}$ via surface diffusion is therefore of outmost importance and should be carefully avoided or taken into account in any FEBID process when determining the fundamental parameters for electron-precursor interactions such as performed in Ref. [30].

\section{MAGNETIC PROPERTIES OF NANOCOMPOSITE CO-C HALL SENSORS}

By using the composition tuning by pulsed exposure in the two adsorbate system $\left(\mathrm{Co}_{2}(\mathrm{CO})_{8}\right.$ and hydrocarbons), we were able to fabricate Hall nano-sensors with a large variation of Cobalt concentrations as shown in Figure 7. The FEB deposits had a nanocomposite structure and superparamagnetic properties intimately linked to the nanoparticle sizes and their relative distance. The magnetic sensitivity was found to decrease exponentially with the metal concentration, fact known as the Giant Hall Effect. ${ }^{29}$

As shown in Table I the Co-C nano-Hall sensors have remarkable magnetic spatial resolution making them suitable for the detection of single paramagnetic beads. ${ }^{29}$ The high flexibility of the FEBID technique allows for on chip or microfluidic channel integration, opening the way for the use of the Co-C Hall-sensors as bio-nanosensors or in Scanning Hall Probe Microscopy applications.

\section{CONCLUSIONS}

Here we have shown the importance of carbon codeposition for all FEBID processes performed in standard high vacuum SEM chambers. By using the two adsorbate concept it was possible to describe the variation of composition with the beam pulse time and to define a pulse duration window when the electron triggered reaction is in the electron-limited regime for one adsorbate and still in the molecule-limited regime for the other one. Unlike standard CVD, the composition can be tuned not only by controlling the molecule flux ratios but also by the $\sigma \times \tau$ ratio of the two adsorbates. We showed that in the particular case when the carbon is provided by surrounding hydrocarbons, the fundamental parameters used for fitting the EDX concentration data depend on the experimental deposition parameters (beam current density, refresh time) and on the deposit size. This work will be used for refining the general theoretical two adsorbate model in order to account for surface gradients and size depended concentration.

The tuning of $\mathrm{Co}-\mathrm{C}$ was used in the fabrication of nanocomposite Hall nanosensors with different active area sizes and metal:matrix ratios. The magnetic properties of the nanocomposite material depend on the Co nanoparticle sizes and their distribution, with a magnetic sensitivity increasing exponentially as the metal content decreases that allowed for the fabrication of nano-sensors with remarkable magnetic spatial resolution.

Acknowledgments: We gratefully acknowledge support from Dr. Martin Jenke and Dr. Stephan Fahlbusch from the Alemnis company for assistance with the gas injection system and dual GIS developments. This work has been carried out with support from the CTI Project No.: 10710.1 PFNM-NM and from EU COST Action: CM0601.

\section{References and Notes}

1. I. Utke and A. Gölzhäuser, Angew. Chem. Int. Ed. 49, 9328 (2010).

2. I. Utke, P. Hoffmann, and J. Melngailis, J. Vac. Sci. Technol. B 26, 1197 (2008)

3. W. F. van Dorp and C. W. Hagen, J. Appl. Phys. 104, 081301 (2008).

4. S. J. Randolph, J. D. Fowlkes, and P. D. Rack, Crit. Rev. Solid State Mater. Sci. 31, 55 (2006).

5. K. Furuya, Science and Technology of Advanced Materials 9, 014110 (2008).

6. M. Tanaka, M. Shimojo, K. Mitsuishi, and K. Furuya, Appl. Phys. A: Mater. Sci. Proc. 78, 543 (2004).

7. D. Spoddig, K. Schindler, P. Roediger, J. Barzola-Quiquia, K. Fritsch, H. Mulders, and P. Esquinazi, Nanotechnology 18, 495202 (2007).

J. Nanosci. Nanotechnol. 11, 7982-7987, 2011 
8. P. Dhakal, G. McMahon, S. Shepard, T. Kirkpatrick, J. I. Oh, and M. J. Naughton, Appl. Phys. Lett. 96, 262511 (2010).

9. C. H. Schwalb, C. Grimm, M. Baranowski, R. Sachser, F. Porrati, H. Reith, P. Das, J. Müller, F. Völklein, A. Kaya, and M. Huth, Sensors 10, 9847 (2010).

10. M. Huth, J. Appl. Phys. 107, 113709 (2010).

11. M. S. Gabureac, L. Bernau, I. Utke, and G. Boero, Nanotechnology 21, 115503 (2010).

12. A. Fernandez-Pacheco, J. M. De Teresa, R. Cordoba, and M. R. Ibarra, J. Phys. D: Appl. Phys. 42, 055005 (2009).

13. M. Takeguchi, M. Shimojo, and K. Furuya, Nanotechnology 16, 1321 (2005).

14. R. R. Kunz and T. M. Mayer, Appl. Phys. Lett. 50, 962 (1987).

15. R. R. Kunz and T. M. Mayer, J. Vac. Sci. Technol. B: Microelectronics and Nanometer Structures 6, 1557 (1988).

16. M.-M. Walz, M. Schirmer, F. Vollnhals, T. Lukasczyk, H.-P. Steinrück, and H. Marbach, Angew. Chem. Int. Ed. 49, 4669 (2010).

17. J. J. Hren, Ultramicroscopy 2, 375 (1979).

18. A. N. Broers, W. W. Molzen, J. J. Cuomo, and N. D. Wittels, Appl. Phys. Lett. 29, 596 (1976).

19. T. Djenizian, L. Santinacci, and P. Schmuki, Appl. Phys. Lett. 78, 2940 (2001).
20. L. Bernau, M. Gabureac, R. Erni, and I. Utke, Angew, Chem. Int. Ed. 49, 8880 (2010).

21. L. Bernau, PhD thesis 4944, Lausanne EPFL (2011).

22. R. A. Marbrow and R. M. Lambert, Surf. Sci. 67, 489 (1977).

23. M. Tanaka, M. Shimojo, M. Takeguchi, K. Mitsuishi, and K. Furuya, J. Cryst. Growth 275, e2361 (2005).

24. T. Lukasczyk, M. Schirmer, H.-P. Steinrück, and H. Marbach, Small 4, 841 (2008).

25. P. C. Cosby, J. Chem. Phys. 98, 7804 (1993).

26. I. Utke, V. Friedli, M. Purrucker, and J. Michler, J. Vac. Sci. Technol., B 25, 2219 (2007).

27. A. Fernández-Pacheco, J. M. De Teresa, A. Szkudlarek, R. Córdoba, M. R. Ibarra, D. Petit, L. O’Brien, H. T. Zeng, E. R. Lewis, D. E. Read, and R. P. Cowburn, Nanotechnology 20, 475704 (2009).

28. T. Bret, S. Mauron, I. Utke, and P. Hoffmann, Microelectron. Eng. 78-79, 300 (2005).

29. M. Gabureac, L. Bernau, I. Utke, A. Fernandez-Pacheco, and J. M. De Teresa, Nanofabrication Using Focused Ion and Electron Beams: Principles and Applications, edited by I. Utke, S. A. Moshkalev, and P. Russell, Oxford University Press, Oxford, New York (2011).

30. J. D. Fowlkes and P. D. Rack, ACS Nano 4, 1619 (2010).

Received: 3 May 2011. Accepted: 13 June 2011. 